Aim of the study: To analyse the incidence of appendiceal neuroendocrine neoplasms in appendectomy specimens and establish the epidemiological and histopathological features, treatment, and clinical course.

Material and methods: Between 2004 and 2013, 975 patients who underwent appendectomy in Ankara Oncology Education and Research Hospital were retrospectively analysed.

Results: Neuroendocrine neoplasm was detected in the nine of $975(0.9 \%)$ patients. Neuroendocrine neoplasms were diagnosed in eight patients by appendectomy, which was performed because of the prediagnosis of acute appendicitis, and in one patient by the suspicious mass detection during surgical procedures that were done in the appendix for a different reason. Eight of the patients' tumours were in the tip of the appendix, and one of the patients' tumours was at the base of appendix. Tumour size in $77.8 \%$ of patients was equal or less than $1 \mathrm{~cm}$, in $22.2 \%$ patients it was $1-2 \mathrm{~cm}$. There was tumour invasion in the muscularis propria layer in four patients, in the serosa layer in three patients, and in the deep mesoappendix in two patients. Patients were followed for a median of 78 months. In the follow-up of patients who were operated because of colon cancer, metachronous colon tumour evolved. This patient died due to progressive disease. Other patients are still disease-free. Conclusions: The diagnosis of neuroendocrine neoplasm is often incidentally done after appendectomy. Tumour size is important in determining the extent of disease and in the selection of the surgical method during operation.

Key words: appendix, appendectomy, incidental, neuroendocrine neoplasms.

Contemp Oncol (Pozn) 2015; 19 (5): 410-413 DOI: $10.5114 /$ wo.2015.56008

\section{Clinical experience in appendiceal neuroendocrine neoplasms}

\author{
Caglar K. Ozcelik ${ }^{1}$, Sevim Turanli ${ }^{2}$, Nazan Bozdogan ${ }^{3}$, Cengiz Dibekoglu
}

'Department of General Surgery, University of Süleyman Demirel, Isparta, Turkey 2Department of General Surgery, Ankara Oncology Education and Research Hospital, Ankara, Turkey

${ }^{3}$ Department of Pathology, Ankara Oncology Education and Research Hospital, Ankara, Turkey

${ }^{4}$ Department of General Surgery, Istanbul Florence Nightingale Hospital, Istanbul, Turkey

\section{Introduction}

Appendix tumours are relatively rare cancers. In the examination of specimens after appendectomy, appendix cancer is observed with $1 \%$ incidence [1]. According to the National Cancer Institute, using the Surveillance, Epidemiology, and End Results (SEER) data, appendix cancer accounts for $0.4 \%$ of all gastrointestinal tumours [2]. Well-differentiated neuroendocrine tumours, traditionally referred to as carcinoid tumours, are the most frequently observed kind [1]. Other cancers of the appendix are mucinous cystic adenocarcinoma, adenocarcinoma, goblet cell carcinoma, lymphoma, and paraganglioma. Generally, in the histopathological examination that is done after appendectomy performed due to acute appendicitis, carcinoid tumours are detected incidentally with a frequency of $0.3-0.9 \%[3,4]$.

In many studies, in appendiceal neuroendocrine neoplasms (NEN) smaller than $1 \mathrm{~cm}$, with invasion up to the subserosa or mesoappendiceal invasion up to $3 \mathrm{~mm}$, and clear surgical margins, no further risk of recurrence is posed after appendectomy and it is considered that there is no risk of metastases $[5,6]$. The intermediate-size group of appendiceal NEN $>1 \mathrm{~cm}$ but $<2 \mathrm{~cm}$ is the group with a less clear situation. However, metastases also seem to occur very rarely in this subgroup, which comprises $5-25 \%$ of all appendiceal NEN, their occurrence has been described in up to 10\% in some series although the largest by Moertel et al. [6] did not report any metastases in this subgroup. Right hemicolectomy is justified only in those rare tumours $1-2 \mathrm{~cm}$ in size but with positive or unclear margins or with deep mesoappendiceal invasion, higher proliferation rate (G2) and/or angioinvasion [5]. However, in tumours larger than $2 \mathrm{~cm}$, the risk of metastasis increases to $25-40 \%[7,8]$. Tumours with a diameter $>2 \mathrm{~cm}$ should be treated by right hemicolectomy.

In this study, the incidence of neuroendocrine neoplasm of the appendix, the epidemiological and histopathological features, the treatment that was used, and the clinical course were evaluated retrospectively.

\section{Material and methods}

In total, 975 patients who were operated in Ankara Oncology Education and Research Hospital between 2004 and 2013 with the diagnosis of acute appendicitis or who were operated for another reason but had incidental detection of a suspicious mass in the appendix and who underwent appendectomy were enrolled in this study. Among these patients, the record of the nine patients whose histopathological examination results showed appendiceal neuroendocrine neoplasm (according to the European Neuroendocrine Tumour Society (ENETS) guidelines 2012) [5] were examined retrospectively. Patients' demographic characteristics, pathological characteristics (tumour 
size, localisation, invasion depth, and immunohistochemical staining characteristics), treatment, and time of following-up were analysed. Study data were evaluated by using descriptive statistical methods (frequency, median).

\section{Results}

In $0.9 \%$ of patients who underwent appendectomy, neuroendocrine neoplasm was detected. The main referral cause of patients to the hospital was abdominal pain, nausea, and vomiting. Symptoms of heat intolerance, sweating, palpitations, and flushing were not detected. In a patient with left colon tumour, abdominal tomography imaging was performed preoperatively. In this patient, no lesion was detected in the appendix localisation with imaging. Neuroendocrine neoplasms were diagnosed in eight patients incidentally by appendectomy performed due to the prediagnosis of acute appendicitis, and in one patient by the examination of the appendectomy specimen that was taken because of the mass detection in the appendix during the surgery of the left colon tumour.

Patient demographics and pathological characteristics are shown in Table 1 . The median age at diagnosis was 32 years (range, $12-57$ years) and the median tumour size was $0.6 \mathrm{~cm}$ (range, $0.2-1.5 \mathrm{~cm}$ ). In all of the seven patients with tumour size equal to or smaller than $1 \mathrm{~cm}$, the tumour showed the distal localisation, in one of the two patients with 1-2-cm tumour size, the tumour was in the proximal appendix, and in the other it was in distal appendix. When the relationship between tumour size and the depth of invasion of tumour considered in four of seven tumours was equal to or smaller than $1 \mathrm{~cm}$, muscularis propria invasion was detected, in two patients there was serosa invasion, and in one patient there was deep mesoappendix invasion. Serosa invasion was detected in one of the two patients with 1-2 cm tumour size, and deep mesoappendix invasion in the other. Right hemicolectomy was applied to two patients because of extensive mesoappendix invasion. During left hemicolectomy because of left colon tumour in one patient, right hemicolectomy was also applied for a suspicious appendix tumour. There was no lymph node involvement or residual tumour of the colon in the specimens of these three patients. Adjuvant chemotherapy was given to colon cancer patients, and the other patients were just followed. Patients were followed for a median of 78 months (range 6-102 months). In the patient operated for colon tumour, metachronous colon tumour developed later. This patient died due to progressive disease. The other patients are still alive and disease free.

\section{Discussion}

Gastrointestinal neuroendocrine tumours (traditionally referred to as carcinoid tumours) are seen most commonly in the small intestine with the frequency of $44.7 \%$, and less frequently in the rectum (19.6\%), appendix (16.7\%), colon (10.6\%), and stomach (7.2\%) [9]. Appendiceal neuroendocrine neoplasms are diagnosed slightly more often in female than in male patients, at an average age of 40-50 years [5, 10, 11]. In our series, median age was 34 years and the tumours were detected at a similar rate in both sexes and were seen with a frequency of $0.9 \%$.

In general, appendiceal NEN are asymptomatic tumours seen in the preoperative period. In a study by Robertson et al. the time between the formation of tumours and the beginning of symptoms was about nine years [12]. Most of the time, acute appendicitis findings developing due to increased intraluminal pressure induced by the tumour clogging appendiceal lumen is the first and unique symptom [13]. Carcinoid syndrome usually develops after liver metastases occur, and it is seen in $10 \%$ of all carcinoid tumours. Major findings are flushing, diarrhoea, skin manifestations (e.g. pellagra), bronchospasm, and progressive congestive heart failure [12]. Generally, since tumour size is small, the sensitivity of the imaging methods is not high. In our study group, except for the patient operated because of colon tumour, in all patients the cause of hospital referral was findings suggestive of acute appendicitis. Carcinoid syndrome was not seen in any patients. Only in one patient, abdominal tomography was performed preoperatively because of colon tumour without revealing a mass lesion in the appendix, and in this patient a mass was suspected during the surgery.

Appendiceal NEN are located most commonly at the tip of the appendix $-75 \%$ of cases, at the base of the appendix in $5 \%$, and in the mid-section in $20 \%$ [14]. In the literature, reported mean tumour size is $6 \mathrm{~mm}$ (range; $0.4-14$ $\mathrm{mm})[5,14]$. In eight patients, the tumour was at the tip of the appendix, and in one patient it was in the base of the appendix; the median tumour size was $6 \mathrm{~mm}$ in this study.

Size of the appendiceal NEN, localisation within the appendix, and the extent of invasion into the mesoappen-

Table 1. Patients' demographics and pathological characteristics

\begin{tabular}{|c|c|c|}
\hline Sex & $\begin{array}{l}\text { Male } \\
\text { Female }\end{array}$ & $\begin{array}{l}5 \text { pts }(55.5 \%) \\
4 \text { pts }(44.5 \%)\end{array}$ \\
\hline Tumour size & $\begin{array}{l}\text { Equal to or less } \\
\text { than } 1 \mathrm{~cm} \\
1-2 \mathrm{~cm}\end{array}$ & $\begin{array}{l}7 \text { pts }(77.8 \%) \\
2 \text { pts }(22.2 \%)\end{array}$ \\
\hline Tumour location & $\begin{array}{l}\text { Tip of the appendix } \\
\text { Base of the } \\
\text { appendix }\end{array}$ & $\begin{array}{l}8 \text { pts }(88.9 \%) \\
1 \text { pts }(11.1 \%)\end{array}$ \\
\hline Tumour invasion & $\begin{array}{l}\text { Muscularis propria } \\
\text { layer } \\
\text { Serosa layer } \\
\text { Deep } \\
\text { mesoappendix }\end{array}$ & $\begin{array}{l}4 \text { pts }(44.5 \%) \\
3 \text { pts }(33.3 \%) \\
2 \text { pts }(22.2 \%)\end{array}$ \\
\hline $\begin{array}{l}\text { Lymph node } \\
\text { involvement }\end{array}$ & No of patients & 0 pts $0(0 \%)$ \\
\hline $\begin{array}{l}\text { Immunohistochemical } \\
\text { examination }\end{array}$ & $\begin{array}{l}\text { Chromogranin } \\
\text { staining } \\
\text { Synaptophysin } \\
\text { staining }\end{array}$ & $\begin{array}{l}9 \text { pts }(100 \%) \\
9 \text { pts }(100 \%)\end{array}$ \\
\hline Grade & Grade 1 & 9 pts $(100 \%)$ \\
\hline Type of surgery & $\begin{array}{l}\text { Appendectomy } \\
\text { Right } \\
\text { hemicolectomy }\end{array}$ & $\begin{array}{l}6 \text { pts }(66.7 \%) \\
3 \text { pts }(33.3 \%)\end{array}$ \\
\hline ENETS stage & $\begin{array}{l}\text { Stage I } \\
\text { Stage II }\end{array}$ & $\begin{array}{l}7 \text { pts }(77.8 \%) \\
2 \text { pts }(22.2 \%)\end{array}$ \\
\hline
\end{tabular}


dix are essential criteria for the therapeutic and follow-up stratification of appendiceal NEN, which are usually well differentiated (G1/2). While infiltration of the appendiceal serosa does not per se seem to be associated with poorer outcome, invasion into the mesoappendix shows a higher rate of lymphatic invasion than in cases without. The depth of invasion beyond $3 \mathrm{~mm}$ has been suggested as reflecting the aggressiveness of the disease [15]. Additional criteria such as a Ki67 index of 3\% or higher (NET-G2) or angioinvasion have been suggested as aiding with decision-making [5]. Since diagnosis is often incidentally made after appendectomy, according to the characteristics of the tumour determined by pathology reports, sometimes second surgery may be required. In a series of 28 patients, all patient with NEN were treated with right hemicolectomy. According to tumour size $(\leq 1 \mathrm{~cm}, 1-2 \mathrm{~cm}$, and $>2 \mathrm{~cm}$ ) in specimens, residual disease detection rates were $3.5 \%$, $18.0 \%$, and $25.0 \%$, respectively. If in these patients the operation decision was taken according only to Ki-67 index, vascular invasion and extensive mesoappendiceal invasion without taking account tumour size, residual disease would be missed in $18 \%$ of appendiceal NEN patients [16]. In our series, right hemicolectomy was applied due to mesoappendix invasion detected in two patients, but residual malignancy was not found in the removed specimens in any of the patients.

The prognosis for the majority of appendiceal NEN is excellent, and the five-year survival of patients is more than $90 \%$. However, the whole cohort including all tumour stages does not show such a favourable prognosis, with the five-year survival rate ranging between 70 and $85 \%$ [17]. In cases with deep mesoappendiceal infiltration or angioinvasion, imaging as outlined above may be performed to rule out any residual disease. All other patients with larger tumours, metastases, or additional risk factors (R1 resection, tumour size $>2 \mathrm{~cm}$ ), should be followed initially after 6 and 12 months postoperatively and then annually. Patients should be monitored by physical examination, 5-HIAA and chromogranin-A levels. Due to the possibility of synchronous or metachronous colorectal carcinoma development at more than 33\%, screening with colonoscopy should be recommended to patients [3, 4, 18]. In our series, one patient developed metachronous colon tumour.

The rate of the distant metastases in all of carcinoid tumours is $4 \%$, and the most common sites of metastasis are the regional lymph nodes and liver [7]. Primary treatment of liver metastases is resection or liver metastasectomy. However, in a Mayo Clinic study, if removal of $90 \%$ of the metastasis is possible, palliative debulking surgery is recommended [19]. In irresectable metastasis, hepatic artery ligation or embolization, percutaneous alcohol injection and high-energy radiofrequency ablation treatments can be applied. If there is irresectable metastasis, medical treatment (octreotide, interferon-alpha, anticancer agents such as capacitabine, dacarbazine, 5-FU, oxaliplatin, and temozolamide, bevacizumab targeting VEGF, and everolimus targeting $\mathrm{mTOR}$ ) and radionuclide therapy should be planned in order to reduce the symptoms [20-23]. In our series of patients, since carcinoid syndrome or metastasis was not detected, additional treatment was not applied to any of the patients.

As a result, since preoperative diagnostic methods are inadequate, neuroendocrine neoplasms of the appendix are often diagnosed by pathological examination. In this disease, which has good long-term prognosis, if tumour size is smaller than $2 \mathrm{~cm}$ and there is no adverse prognostic factor, appendectomy is sufficient in the treatment. If tumour size is greater than $2 \mathrm{~cm}$ and there is tumour invasion beneath the serosa of the appendix or positive or unclear margins or with deep mesoappendiceal invasion, the presence of higher proliferation rate (G2), and/or angioinvasion after appendectomy, right hemicolectomy should be applied. Patients should be monitored periodically for recurrence and synchronous and/or metachronous colorectal cancer development.

The authors declare conflicts of interest.

This submission was presented in the European Society for Surgical Research 2013

\section{References}

1. McCusker ME, Coté TR, Clegg LX, Sobin LH. Primary malignant neoplasm of the appendix: a population-based study from the surveillance, epidemiology and end-results program, 1973-1998. Cancer 2002; 94: 3307-12.

2. Ruoff C, Hanna L, Zhi W, Shahzad G, Gotlieb V, Saif MW. Cancers of the Appendix: Review of the Literatures. ISRN Oncol 2011; 2011: 728579.

3. Tchana-Sato V, Detry O, Detroz B, et al. Carcinoid tumour of the appendix: a consecutive series from 1237 appendectomies. World J Gastroenterol 2006; 12: 6699-701.

4. Groth SS, Virnig BA, Al-Refaie WB, Jarosek SL, Jensen EH, Tuttle TM. Appendiceal carcinoid tumors: predictors of lymph node metastasis and the impact of right hemicolectomy on survival. J Surg Oncol 2011; 103: 39-45.

5. Pape UF, Perren A, Niederle B, et al. ENETS Consensus Guidelines for the management of patients with neuroendocrine neoplasms from the jejuno-ileum and the appendix including Goblet Cell Carcinomas. Neuroendocrinology 2012; 95: 135-56.

6. Moertel CG, Weiland LH, Nagorney DM, Dockerty MB. Carcinoid tumor of the appendix: treatment and prognosis. N Engl J Med 1987; 317: 1699-701.

7. MacGillivray DC, Synder DA, Drucker W, ReMine SG. Carcinoid tumors: the relationship between clinical presentation and the extent of disease. Surgery 1991; 110: 68-72.

8. In't Hof KH1, van der Wal HC, Kazemier G, Lange JF. Carcinoid tumour of the appendix: an analysis of 1,485 consecutive emergency appendectomies. J Gastrointest Surg 2008; 12: 1436-8.

9. Maggard MA, O'Connell JB, Ko CY. Updated population-based review of carcinoid tumors. Annals of Surgery 2004; 240: 117-22.

10. Yao JC, Hassan M, Phan A, et al. One hundred years after 'carcinoid': epidemiology of and prognostic factors for neuroendocrine tumors in 35,825 cases in the United States. J Clin Oncol 2008; 26: 3063-72.

11. Hauso O, Gustafsson BI, Kidd M, Waldum HL, Drozdov I, Chan AK, Modlin IM. Neuroendocrine tumor epidemiology: contrasting Norway and North America. Cancer 2008; 113: 2655-64.

12. Robertson RG, Geiger WJ, Davis NB. Carcinoid tumors. American Family Physician 2006; 74: 429-34.

13. Coşkun H, Bostanci O, Dilege ME, Mihmanli M, Yilmaz B, Akgün I, Yildirim S. Carcinoid tumors of appendix: treatment and outcome. Ulus Travma Acil Cerrahi Derg 2006; 12: 150-4. 
14. Prommegger R, Obrist P, Ensinger C, Profanter C, Mittermair R, Hager J. Retrospective evaluation of carcinoid tumors of he appendix in children. World J Surg 2002; 1489-92.

15. Rindi G, Klöppel G, Couvelard A, et al. TNM staging of midgut and hindgut (neuro)endocrine tumors: a consensus proposal including a grading system. Virchows Arch 2007; 451: 757-62.

16. Grozinsky-Glasberg S, Alexandraki KI, Barak D, Doviner V, Reissman P, Kaltsas GA, Gross DJ. Current size criteria for the management of neuroendocrine tumors of the appendix: are they valid? Clinical experience and review of the literature. Neuroendocrinology 2013; 98: 31-7.

17. García-Carbonero R, Capdevila J, Crespo-Herrero G, et al. Incidence, patterns of care and prognostic factors for outcome of gastroenteropancreatic neuroendocrine tumors (GEP-NETs): results from the National Cancer Registry of Spain (RGETNE). Ann Oncol 2010; 21: $1794-803$

18. Modlin IM, Lye KD, Kidd M. A 5-decade analysis of 13715 carcinoid tumors. Cancer 2003; 97: 934-59.

19. Kvols LK. Metastatic carcinoid tumors and the carcinoid syndrome. A selective review of chemotherapy and hormonal therapy. Am J Med 1986; 81: 49-55.

20. Cappell MS. The pathophysiology, clinical presentation, and diagnosis of colon cancer and adenomatous polyps. Med Clin North Am 2005; 89: 1-42.

21. Kulke MH, Mayer RJ. Carcinoid tumors. N Engl J Med 1999; 340: 858-68.

22. Oberg K, Eriksson B. The role of interferon in the management of carcinoid tumors, Acta Oncol 1991; 30: 519-22.

23. Paulson AS, Bergsland EK. Systemic therapy for advanced carcinoid tumors: where do we go from here? I Natl Compr Canc Netw 2012; 10: 785-93.

\section{Address for correspondence}

\section{Sevim Turanli}

Department of General Surgery

Ankara Oncology Education and Research Hospital

06180 Ankara, Turkey

e-mail: turanlisevim@hotmail.com

Submitted: 18.01.2014

Accepted: $\quad 6.08 .2014$ 